\title{
Relación entre pastura (Axonopus catarinensi), lapso de suplementación y crecimiento de vaquillonas en sistema silvopastoril
}

\author{
Pantiu, A.J., ${ }^{1}$ Capellari, A. ${ }^{1}$; Giménez L.I. ${ }^{2}$ \\ ${ }^{1}$ Facultad de Ciencias Veterinarias, Universidad Nacional del Nordeste (UNNE), Cabral 2139, Corrientes (3400), \\ Argentina. ${ }^{2}$ Facultad de Ciencias Agrarias (UNNE). E-mail: apantiu@gmail.com
}

\begin{abstract}
Resumen
Pantiu, A.J.; Capellari, A.; Giménez L.I.: Relación entre pastura (Axonopus catarinensi), lapso de suplementación y crecimiento de vaquillonas en sistema silvopastoril. Rev. vet. 26: 1, 22-26, 2015. El objetivo del trabajo fue determinar la relación entre el crecimiento de vaquillonas, el lapso de suplementación y la disponibilidad de pastura en un sistema silvopastoril. El diseño experimental fue completamente aleatorizado con dos tratamientos $(\mathrm{T} 1, \mathrm{~T} 2)$ y cuatro repeticiones. La unidad experimental fue cada lote, compuesto por 7 vaquillonas (2,8 vaq/ha). En T1 los animales recibieron suplementación formulada para mantener una ganancia diaria de 0,6 kg/día, durante los nueve meses que duró el ensayo. En T2 los animales recibieron la misma suplementación que T1 durante cuatro meses en el invierno, y solo pastura el resto del periodo. La suplementación estuvo compuesta por $56 \%$ de maíz y $44 \%$ de expeller de algodón al $0,83 \%$ del peso vivo, disponiendo de pasto jesuita gigante (Axonopus catarinensis) a voluntad. Cada 28 a 32 días se estableció: peso vivo, ganancia total y diaria de peso, alzada a la cruz y grupa, longitud corporal y perímetro torácico. Para evaluar la pastura se utilizó el método del disco y la calidad fue medida en la porción consumida. Con los datos obtenidos se realizó un análisis estadístico descriptivo. Las variables del componente forrajero y animal se analizaron en un contexto de modelos lineales generales y mixtos para medidas repetidas en el tiempo, donde las variables clasificatorias fueron tratamiento, parcela y tiempo. No se obtuvieron diferencias estadísticamente significativas entre los tratamientos, por lo que se informa un análisis de correlación con el método de Pearson. El peso se asoció fuertemente con el perímetro torácico $(\mathrm{r}=0,95 ; \mathrm{p}<0,001)$, alzada a la cruz $(\mathrm{r}=0,79 ; \mathrm{p}<0,001)$ y grupa $(\mathrm{r}=0,79 ; \mathrm{p}<0,001)$. La proteína bruta $(\mathrm{r}=-0,46 ; \mathrm{p}<0,0001)$ y la disponibilidad $(\mathrm{r}=0,76 ; \mathrm{p}<0,0001)$ se relacionaron con el peso y la disponibilidad con alzada a la cruz $(\mathrm{r}=0,76 ; \mathrm{p}<0,001)$, grupa $(\mathrm{r}=0,74 ; \mathrm{p}<0,001)$ y perímetro torácico $(\mathrm{r}=0,82 ; \mathrm{p}<0,001)$. Se concluye que la cantidad de forraje disponible se correlaciona con la respuesta animal, ya que una mayor cantidad de materia seca se manifiesta en un mayor peso y tamaño corporal.
\end{abstract}

Palabras clave: vaquillona, crecimiento, calidad de pastura, disponibilidad, sistema silvopastoril.

\begin{abstract}
Pantiu, A.J.; Capellari, A.; Giménez L.I.: Relationship among pasture (Axonopus catarinensis), lapse of supplementation, and growth of heifers on silvopastoral system. Rev. vet. 26: 1, 22-26, 2015. The aim of this study was to determine the relationship among growth of heifers, lapse of supplementation, and availability of a pasture (Axonopus caterinensis) on a silvopastoral system. Experimental design was randomly carried out with two treatments (T1, T2) and four repetitions. In T1 animals received feeding supplementation during the nine months that the test took place and in $\mathrm{T} 2$ they received the same supplementation as in T1 for four months during winter but were fed only with pasture for the rest of the period. Supplementation consisted of $56 \%$ maize and $44 \%$ cotton expeller to $0.83 \%$ of liveweight, with Axonopus catarinensis available at will. Every 28 to 32 days animals were measured for liveweight, total and daily weight gain, height at withers and at rumps, body length, and thoracic circumference. Pasture sampling was performed by means of the disk method and quality was measured in the consumed pasture portion. A correlation with Pearson method was conducted, with no significant differences between the treatments. Weight was sig-
\end{abstract}


nificantly correlated with the thoracic circumference $(r=0.95 ; \mathrm{p}<0.001),(\mathrm{r}=0.79 ; \mathrm{p}<0.001)$, $(\mathrm{r}=0.79 ; \mathrm{p}<0.001)$. Raw protein $(\mathrm{r}=-0.46 ; \mathrm{p}<0.0001)$ and availability $(\mathrm{r}=0.76 ; \mathrm{p}<0.0001)$ were significantly correlated with weight, as well as availability and height at withers $(\mathrm{r}=0.76$; $\mathrm{p}<0.001)$, height at rumps $(\mathrm{r}=0.74 ; \mathrm{p}<0.001)$ and thoracic circumference $(\mathrm{r}=0.81 ; \mathrm{p}<0.001)$. It is concluded that the amount of available forage is a determinant for animals' response, since a larger quantity of dry matter means greater body weight.

Key words: heifer, growth, pasture quality, availability, silvopastoral system.

\section{INTRODUCCIÓN}

En Argentina las pasturas constituyen la fuente de alimentación más económica para los rumiantes, por lo cual es fundamental potenciar su productividad y la eficiencia conque el forraje es cosechado por los animales $\mathrm{y}$ trasformado en producto final ${ }^{1}$.

La comparación de la calidad de los alimentos podría ser realizada a través de la respuesta animal (ganancia diaria de peso vivo o producción de carne) en condiciones no limitantes ${ }^{21}$. En un sistema de producción basado en forrajes, se podría considerar que la mejor pastura en cuanto calidad es aquélla con la que se obtiene mejor respuesta productiva, pero en realidad no se sabe a ciencia cierta hasta qué punto ésta respuesta está relacionada con factores del forraje (calidad y cantidad) y no con otros factores (potencial animal, sanidad, clima y suplementos) ${ }^{1}$.

En el nordeste argentino las pasturas presentan altos niveles de acumulación de forraje en el período primavera-verano-otoño y escaso o nulo crecimiento invernal, produciéndose un alto grado de variación en la concentración de los nutrientes, lo que en consecuencia condicionan las respuestas de las vaquillonas de recría ${ }^{17}$.

Con el objetivo de maximizar el potencial productivo de los sistemas ganaderos en Misiones (Argentina), actualmente se observa una gran adopción de los sistemas silvopastoriles (SSP) ${ }^{4}$, los cuales constituyen logros científicos desarrollados a partir de los resultados de investigaciones que se vienen realizando desde la década de $1980^{7}$.

La renovación e introducción de pastos apropiados, adaptados a las condiciones edafo-climáticas locales, junto a la incorporación estratégica de especies arbóreas, parece ser una alternativa tecnológica que puede contribuir a mejorar la producción bovina ${ }^{4}$. Las interacciones entre los distintos componentes de un SSP (árbol, pastura, animal, suelo y agua) son dinámicas y complejas, con relaciones activas que generan cambios entre sus componentes y la necesidad de un manejo intensivo. El conocimiento de los diferentes grados de interacción y su manejo, son claves para la utilización racional y sustentable del sistema, siendo algunos de ellos, todavía una incógnita ${ }^{16}$.

La producción ganadera sobre pasturas depende en gran parte de la cantidad y calidad del forraje producido, la capacidad del animal para cosecharlo y de las pautas de pastoreo aplicadas para facilitar un mayor consumo y utilización eficiente del mismo ${ }^{5}$. Si la cantidad de forraje presente no limita el consumo del animal, será el valor nutritivo el que determinará la cantidad de producto animal obtenido ${ }^{1}$.

El objetivo del estudio fue determinar la relación entre las variables de crecimiento de las vaquillonas de reposición y entre éstas y las variables de cantidad y calidad de la pastura Axonopus catarinensis, bajo dos tratamientos de alimentación en un sistema silvopastoril.

\section{MATERIAL Y MÉTODOS}

El ensayo se realizó en el establecimiento El Molino de Plantar S.A., localizado en Puerto Esperanza, Provincia de Misiones, Argentina (26.01'26.41' $\mathrm{S}$ y $54^{\circ} 34^{`} 32.51^{\prime \prime O}$ ). El clima de la región es subtropical húmedo, con temperaturas medias anuales del orden de $\operatorname{los} 21,5^{\circ} \mathrm{C}$, presencia de heladas invernales y precipitaciones medias anuales de $1.800 \mathrm{~mm}^{8}$.

Se trabajó en un sistema silvopastoril constituido por Pinus elliottii var elliottii x Pinus caribaea var Hondurensis F2 (pino híbrido F2) de 7 años de edad, a una densidad de 400 árboles/ha (componente forestal); Axonopus catarinensis (pasto jesuita gigante) de 5 años de edad (componente forrajero) y cincuenta y seis vaquillonas para recría tipo Braford con una edad promedio de 10 meses (componente animal). La longitud de copa viva/ha se mantuvo en $3.334,5 \pm 89,6 \mathrm{~m}$ lineales

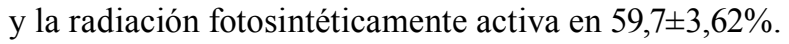

El diseño experimental fue completamente aleatorizado con dos tratamientos de suplementación (T1 y T2) y cuatro repeticiones (lotes de pastoreo de 2,5 ha cada uno). La unidad experimental fue cada lote, que alojó 7 vaquillonas (2,8 vaq/ha). En T1 los animales recibieron suplementación durante los nueve meses que duró el ensayo, formulada para mantener una ganancia diaria de $0,6 \mathrm{~kg} / \mathrm{día}$; para llevarlos de $160 \mathrm{~kg}$ a $320 \mathrm{~kg}$ de peso vivo. En T2 los animales recibieron la misma suplementación que T1 durante cuatro meses en el invierno, y el resto del periodo consumieron solo pastura.

La suplementación estuvo compuesta por $56 \%$ de maíz y $44 \%$ de expeller de algodón; a razón de 0,80 y $0,53 \mathrm{~kg}$ materia seca/animal/día promedio, respectivamente. El nivel de suplementación promedio fue del $0,83 \%$ del PV, disponiendo de pasto jesuita a voluntad. En la Tabla 1 se consigna el resultado del análisis de los 
Tabla 1. Valores nutricionales del maíz y expeller de algodón.

\begin{tabular}{lcc}
\hline variable & maíz & expeller \\
\hline fibra detergente neutro (\%) & 31,34 & 38,67 \\
fibra detergente ácido (\%) & 3,79 & 22,90 \\
total nutrientes digestibles (\%) & 85,23 & 74,27 \\
energía digestible (Mcal/kg MS) & 3,76 & 3,28 \\
proteína bruta (\%) & 11,01 & 45,42 \\
digestibilidad in vitro MS (\%) & 85,54 & 68,67 \\
\hline
\end{tabular}

Análisis realizados en el Laboratorio de Forrajes del Área de Producción Animal, EEA INTA Rafaela. MS: materia seca.

componentes nutricionales de los suplementos empleados a lo largo de la experiencia.

El método de pastoreo fue rotativo y el criterio utilizado para realizar las rotaciones de los animales, fue por medio de la evaluación del remanente no pastoreado. Para ello, se caminó en zigzag en el potrero tomando como referencia las varillas de los alambrados, contando los pasos y, mediante el uso de un contador, se registraron los sectores no pastoreados. De esta manera, cuando una de las repeticiones de un tratamiento alcanzó un máximo de $10 \%$ de remanente, se procedió a la rotación de los animales de todas las parcelas del mismo tratamiento. Estas mediciones se realizaron druante los días lunes, miércoles y viernes.

La toma de muestra para la evaluación de cantidad y calidad de la pastura se efectuó en cada rotación. El muestreo se realizó usando el método del pasturómetro de altura ${ }^{20}$. Antes de que los animales ingresaran al área experimental (pre-pastoreo), se procedió a la medición de 50 puntos al azar en cada una de las franjas de pastoreo. Posteriormente, con el rango total de alturas medidas con el disco, se realizó una división en 3 sub-rangos (alta, media y baja altura) para cada potrero. Luego se midió la altura utilizando el disco y la altura extendida de las hojas y pseudo-tallos de los cuatro macollos más altos, tomándose una muestra por corte de círculos de $45 \mathrm{~cm}$ al ras del suelo de cada sub-rango en cada una de las franjas de pastoreo de cada tratamiento.

Las muestras fueron embolsadas sin doblarlas y llevadas al laboratorio donde el material de cada aro fue pesado en fresco y secado en estufa a $60^{\circ} \mathrm{C}$ hasta peso constante, para la obtención de cantidad de materia seca. En base a estos datos se estableció una curva de regresión entre la altura de la pastura medida con el disco y las variables de la pastura (biomasa, altura extendida de macollos y pseudo-tallos), para estimar la disponibilidad pre-pastoreo de materia seca por hectárea de forraje ( $\mathrm{kg}$ de $\mathrm{MS} / \mathrm{ha})$.

Posteriormente, luego de la rotación (pos-pastoreo) se realizó el mismo número y tipo de mediciones (excepto altura de pseudo-tallo) que en el pre-pastoreo, en los sitios localizados de evaluación, sumado a la medición de los diámetros de los manchones no pastoreados para determinar la superficie de los mismos. Con estos datos se estableció otra curva de regresión para estimar disponibilidad pos-pastoreo en $\mathrm{kg}$ de MS/ha. Mediante la diferencia entre la disponibilidad de materia seca pre y pos-pastoreo se estimó el consumo de materia seca.

La calidad se midió en la porción estimada de consumo de la pastura. Para ello, se envió al laboratorio una muestra compuesta, agrupando las de cada franja, donde se obtuvieron las siguientes variables: contenido de proteína bruta $(\mathrm{PB})$, fibra detergente neutro (FDN), fibra detergente ácido (FDA), total de nutrientes digestibles (TND) ${ }^{11}$, estimación de energía digestible (ED) ${ }^{14}$ y digestibilidad de la MS (DMS).

Cada 28 a 32 días se efectuaron pesajes individuales, a partir de los cuales se calculó la ganancia de peso diario (GPD). Con una cinta métrica metálica se midió altura a la cruz (AC) y grupa (AG), longitud corporal (LC) y perímetro torácico $(\mathrm{PT})^{22}$.

Con los datos obtenidos se realizó un análisis estadístico descriptivo, las variables de los componentes forrajero y animal se analizaron en un contexto de modelos lineales generales y mixtos para medidas repetidas en el tiempo, donde las variables clasificatorias fueron tratamiento, parcela y tiempo. Posteriormente, se realizó un análisis de correlaciones entre las variables del componente animal y entre éstas y las variables del componente forrajero, mediante el método de Pearson. Se utilizó el paquete estadístico InfoStat ${ }^{3}$.

\section{RESULTADOS Y DISCUSIÓN}

En la Tabla 2 se consignan los promedios, desvíos estándares, máximos y mínimos obtenidos de las variables de los componentes animal y forrajero del ensayo. Efectuado el análisis de correlación entre las variables

Tabla 2. Valores obtenidos en las vaquillonas bajo ensayo $(n=36)$ y evaluación cuali-cuantitativa de la pastura.

\begin{tabular}{lcccc}
\hline variable & $\mathrm{x}$ & $\mathrm{DE}$ & mínimo & máximo \\
\hline PV $(\mathrm{kg})$ & 211,33 & 34,76 & 149,43 & 273,17 \\
GDP $(\mathrm{kg})$ & 0,431 & 0,11 & 0,22 & 0,64 \\
AC $(\mathrm{m})$ & 1,03 & 0,10 & 0,87 & 1,22 \\
AG $(\mathrm{m})$ & 1,03 & 0,14 & 0,81 & 1,21 \\
PT (m) & 1,40 & 0,09 & 1,25 & 1,59 \\
LC (m) & 0,85 & 0,05 & 0,78 & 1,05 \\
Disp (kg MS/ha) & 4.708 & 1.364 & 3.058 & 8.029 \\
FDN (\%) & 66,48 & 2,42 & 61,93 & 72,87 \\
FDA (\%) & 33,98 & 3,11 & 28,72 & 42,93 \\
TND (\%) & 65,43 & 2,48 & 58,32 & 69,63 \\
ED (Mcal/kg MS) & 2,88 & 0,11 & 2,57 & 3,07 \\
PB (\%) & 13,46 & 1,92 & 8,35 & 17,04 \\
DMS (\%) & 46,29 & 3,76 & 36,36 & 53,42 \\
\hline
\end{tabular}

Х: media aritmática, DE: desvío estándar, PV: peso vivo, GDP: ganancia diaria de peso, AC: alzada a la cruz, AG: alzada a la grupa, PT: perímetro torácico, LC: longitud corporal, Disp: disponibilidad de pastura, FDN: fibra detergente neutro, FDA: fibra detergente ácido, TND: total de nutrientes digestibles, ED: energía digestible, PB: proteína bruta, DMS: digestibilidad de la materia seca. 
Tabla 3. Correlación (Pearson) entre los parámetros del crecimiento de los animales y la composición de la pastura.

\begin{tabular}{|c|c|c|c|c|c|}
\hline variable & PV & $\mathrm{AC}$ & AG & PT & LC \\
\hline PV & 1,000 & $\begin{array}{c}0,791 \\
\mathrm{p}<0,0001\end{array}$ & $\begin{array}{c}0,797 \\
\mathrm{p}<0,0001\end{array}$ & $\begin{array}{c}0,955 \\
p<0,0001\end{array}$ & $\begin{array}{c}0,227 \\
p=0,0545\end{array}$ \\
\hline $\mathrm{AC}$ & $\begin{array}{c}0,790 \\
\mathrm{p}<0,0001\end{array}$ & 1,000 & $\begin{array}{c}0,983 \\
\mathrm{p}<0,0001\end{array}$ & $\begin{array}{c}0,869 \\
p<0,0001\end{array}$ & $\begin{array}{c}0,319 \\
p=0,0062\end{array}$ \\
\hline $\mathrm{AG}$ & $\begin{array}{c}0,797 \\
\mathrm{p}<0,0001\end{array}$ & $\begin{array}{c}0,983 \\
\mathrm{p}<0,0001\end{array}$ & 1,000 & $\begin{array}{c}0,862 \\
p<0,0001\end{array}$ & $\begin{array}{c}0,259 \\
\mathrm{p}=0,027\end{array}$ \\
\hline PT & $\begin{array}{c}0,955 \\
\mathrm{p}<0,0001\end{array}$ & $\begin{array}{c}0,869 \\
p<0,0001\end{array}$ & $\begin{array}{c}0,862 \\
p<0,0001\end{array}$ & 1,000 & $\begin{array}{c}0,279 \\
p=0,0173\end{array}$ \\
\hline LC & $\begin{array}{c}0,227 \\
p=0,054\end{array}$ & $\begin{array}{c}0,319 \\
p=0,006\end{array}$ & $\begin{array}{c}0,259 \\
p=0,027\end{array}$ & $\begin{array}{c}0,279 \\
p=0,017\end{array}$ & 1,000 \\
\hline Disp & $\begin{array}{c}0,756 \\
\mathrm{p}<0,0001\end{array}$ & $\begin{array}{c}0,767 \\
\mathrm{p}<0,0001\end{array}$ & $\begin{array}{c}0,747 \\
\mathrm{p}<0,0001\end{array}$ & $\begin{array}{c}0,822 \\
p<0,0001\end{array}$ & $\begin{array}{c}0,24 \\
\mathrm{p}=0,042\end{array}$ \\
\hline FDN & $\begin{array}{c}-0,346 \\
p=0,002\end{array}$ & $\begin{array}{l}-0,259 \\
p=0,027\end{array}$ & $\begin{array}{l}-0,298 \\
p=0,011\end{array}$ & $\begin{array}{l}-0,332 \\
p=0,004\end{array}$ & $\begin{array}{c}0,05 \\
\mathrm{p}=0,697\end{array}$ \\
\hline FDA & $\begin{array}{c}-0,147 \\
\mathrm{p}=0,216\end{array}$ & $\begin{array}{l}-0,130 \\
\mathrm{p}=0,275\end{array}$ & $\begin{array}{c}-0,212 \\
\mathrm{p}=0,073\end{array}$ & $\begin{array}{c}-0,140 \\
\mathrm{p}=0,238\end{array}$ & $\begin{array}{c}0,21 \\
\mathrm{p}=0,073\end{array}$ \\
\hline TND & $\begin{array}{c}0,148 \\
\mathrm{p}=0,211\end{array}$ & $\begin{array}{c}0,124 \\
p=0,297\end{array}$ & $\begin{array}{c}0,210 \\
\mathrm{p}=0,075\end{array}$ & $\begin{array}{c}0,144 \\
p=0,2267\end{array}$ & $\begin{array}{c}-0,21 \\
\mathrm{p}=0,080\end{array}$ \\
\hline ED & $\begin{array}{c}0,168 \\
\mathrm{p}=0,158\end{array}$ & $\begin{array}{c}0,166 \\
\mathrm{p}=0,163\end{array}$ & $\begin{array}{c}0,236 \\
\mathrm{p}=0,045\end{array}$ & $\begin{array}{c}0,160 \\
\mathrm{p}=0,178\end{array}$ & $\begin{array}{c}-0,18 \\
\mathrm{p}=0,122\end{array}$ \\
\hline PB & $\begin{array}{c}-0,464 \\
\mathrm{p}<0,0001\end{array}$ & $\begin{array}{c}-0,437 \\
\mathrm{p}<0,0001\end{array}$ & $\begin{array}{c}-0,408 \\
p=0,0004\end{array}$ & $\begin{array}{c}-0,489 \\
p<0,0001\end{array}$ & $\begin{array}{c}-0,03 \\
\mathrm{p}=0,811\end{array}$ \\
\hline DMS & $\begin{array}{c}0,003 \\
p=0,979\end{array}$ & $\begin{array}{c}0,109 \\
p=0,361\end{array}$ & $\begin{array}{c}0,106 \\
\mathrm{p}=0,373\end{array}$ & $\begin{array}{c}0,047 \\
p=0,693\end{array}$ & $\begin{array}{c}0,20 \\
\mathrm{p}=0,095\end{array}$ \\
\hline
\end{tabular}

PV: peso vivo, AC: alzada a la cruz, AG: alzada a la grupa, PT: perímetro torácico, LC: longitud corporal, FDN: fibra detergente neutro, FDA: fibra detergente ácido, TND: total de nutrientes digestibles, ED: energía digestible, PB: proteína bruta, DMS: digestibilidad de la materia seca, p: significación estadística.

de crecimiento animal y entre estas con las variables de la pastura, fue posible determinar que no hubo diferencia significativa entre los tratamientos, por lo que a continuación se presentan las correlaciones sin tener en cuenta el tratamiento (Tabla 3).

Se observaron valores altamente significativos de $r=0,955 ; 0,791$ y $0,797(p<0,0001)$ entre el peso vivo con el perímetro torácico, alzada a la cruz y alzada a la grupa respectivamente, lo cual indica que estas variables estuvieron altamente asociadas. Ello confirma que un cambio en el PV del animal puede detectarse por un cambio en PT, AC y AG. Similares resultados fueron expuestos por otros autores, quienes sostuvieron que existe una alta correlación entre el peso vivo y algunas medidas corporales, ya que todo aumento de peso va acompañado de un aumento del tamaño $2,9,24$.

En particular, la relación entre peso vivo y perímetro torácico, fue la correlación más alta de las aquí estudiadas; valor similar fue encontrado por otros autores ${ }^{6}$, 13, 18, 19, 25, 26 . Esto indicaría que los animales más "profundos" son también los más "pesados" (Figura 1). Aunque el coeficiente de correlación fue menor entre el peso vivo y alzada, la relación entre estas variables sigue siendo alta, implicándose que los animales más altos son también los más pesados.

Por otra parte, se estableció que no hubo relaciones estadísticamente significativas entre las variables de crecimiento de las vaquillonas con las variables FDN, FDA, TND, ED y DMS. En cambio PB y disponibilidad forrajera arrojaron como resultado un grado de correlación altamente significativo con PV y a su vez, la disponibilidad también se correlacionó significativamente con AC, AG y PT (Tabla 3). Es decir, un aumento en la disponibilidad forrajera se traduce en un aumento del peso vivo, altura y de perímetro torácico en las vaquillonas, en coincidencia con reportes de otros autores ${ }^{10,23}$.

Además, existió correlación negativa entre PV y $\mathrm{PB}$, lo cual es coherente ya que a menor peso mayores

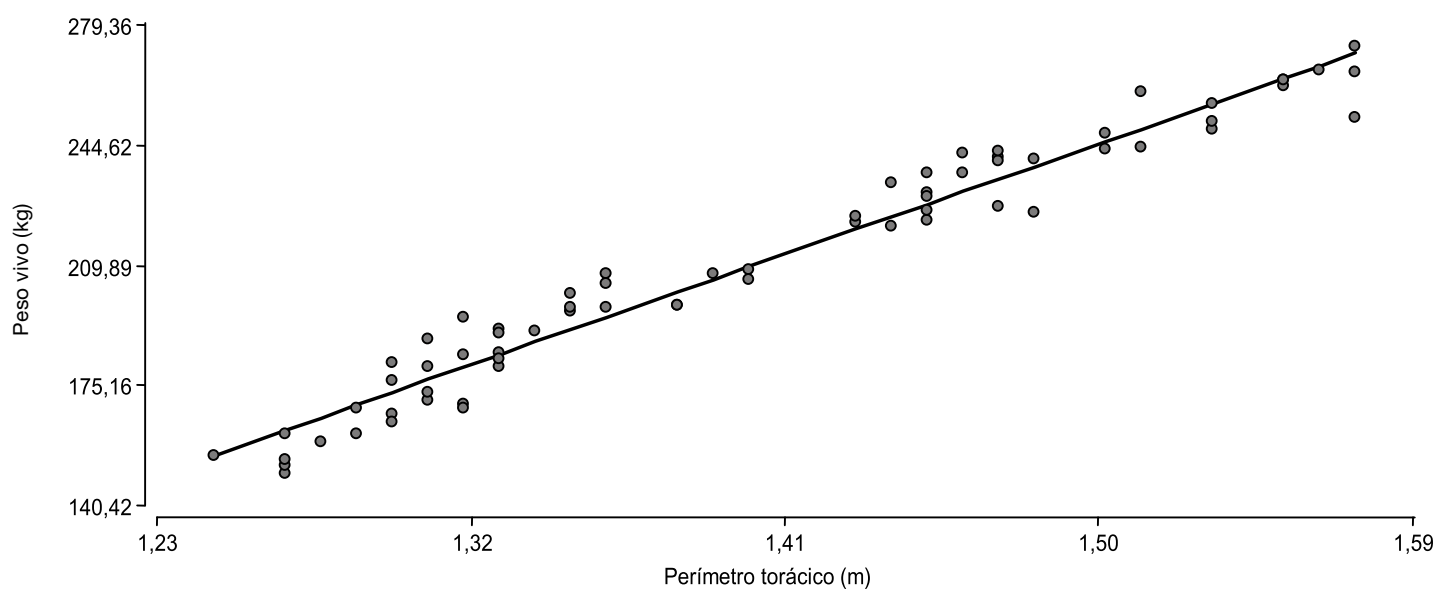

Figura 1. Relación entre peso vivo y perímetro torácico de las vaquillonas. 
son los requerimientos de proteína. Similares resultados fueron publicados por otros investigadores ${ }^{12,15}$.

En conclusión, el crecimiento -traducido en un aumento del peso vivo- se acompaña con el aumento del tamaño corporal. A su vez, una mayor cantidad de materia seca se manifiesta en un mayor peso corporal y los requerimientos de proteína son más altos en los animales en crecimiento, disminuyendo sus necesidades en la dieta a medida que aumenta el peso vivo.

\section{REFERENCIAS}

1. Cangiano C, Brizuela MA. 2011. Producción animal en pastoreo, Publ. INTA, Buenos Aires, p. 511.

2. Di Marco ON. 1998. Crecimiento de vacunos para carne, Ed. Centro del Copiado, Mar del Plata, Argentina, p. 246.

3. Di Rienzo JA, Casanoves F, Balzarini MG, González L, Tablada M, Robledo CW. 2008. InfoStat, versión 2008, Grupo InfoStat, FCA, Universidad Nacional de Córdoba, Argentina.

4. Fassola HE, Lacorte SM, Pachas AN, Goldfarb C, Esquivel J, Colcombet L, Crechi EH, Keller A, Barth SR. 2009. Los sistemas silvopastoriles en la región subtropical del nordeste argentino. Anales XIII Congreso Forestal Mundial, Buenos Aires, Argentina, p. 1-8.

5. Galli JR. 1997. Las pasturas como fuente de alimentación de rumiantes. En: Producción animal en pastoreo, Ed. INTA - Estación Experimental Agropecuaria Balcarce, Argentina, p. 27-40.

6. Garro JM, Rosales LR. 1996. Relación entre el peso corporal y el perímetro torácico en el ganado cebuino en crecimiento en Costa Rica. Agronomía Costarricense 20: 113-123.

7. Iglesias JM, Simón L, Lamela L, Hernández D, Hernández I, Milera M, Castillo E, Sánchez T. 2006. Sistemas agroforestales en Cuba: algunos aspectos de la producción animal. Pastos y Forrajes 29: 1-12.

8. Instituto Nacional de Tecnología Agropecuaria INTA. Plan de Tecnología Regional 2006-2008, Centro Regional Misiones, Argentina, p. 48.

9. Khalil R, Vaccaro L. 2002. Peso y mediciones corporales en vacas de doble propósito: su interacción y asociación con valor genético para tres características productivas. Zoot Trop 20: 11-30.

10. Livas CF. 2000. Engorda de ganado bovino en condiciones de trópico. Anales XXIV Congreso Nacional de Buiatría, Guadalajara, México, p. 71-75.

11. Lofgreen GP. 1951. The use of digestible energy in the evaluation of feeds. J Anim Sci 10: 344-351.

12. MacLoughlin RJ. 2007. Proteína metabolizable y nutrición de bovinos para carne. En: Bovinos para carne; Sección: Fisiología digestiva y manejo del alimento, $N^{\circ} 112$, http://www.produccion-animal.com.ar
13. Mejia N, Velázquez C, Tewolde A. 1996. Predicción del peso vivo en ganado bovino a partir de mediciones corporales. Agronomia mesoamericana 7: 73-76.

14. National Research Council (NRC). 1987. Predicting feed intake of food producing animals, National Academy Press, Washington DC, 96 p.

15. Obispo NE, Pares P, Hidalgo C, Palma J, Godoy S. 2001. Consumo de forraje y ganancia diaria de peso en bovinos de carne en crecimiento suplementados con fuentes proteicas. Zoot Trop 19: 423-442.

16. Ojeda PA, Restrepo JM, Villada DE, Gallego JC. 2003. Sistemas silvopastoriles, una opción para el manejo sustentable de la ganadería, Ed. Fundación para la Investigación y Desarrollo Agrícola (FIDAR), Cali, Colombia, 54 p.

17. Peruchena CO. 2012. Los forrajes y la alimentación para intensificar la producción de carne del norte argentino. http://www.produccion-animal.com.

18. Rathi SS, Balalne DS, Singh BN, Chinkara BS. 1980. Estimations of body weight through measurements in different genetic groups of cattle. Ind J Sci 33: 410-411.

19. Rao GN, Nagarcenkar R. 1979. A note on the interrelationship among body measures in cross breed cattle. Ind $J$ Anim Sci 50: 877-878.

20. Rayburn EB. 1997. An acrylic plastic weight plate for estimating forage yield, West Virginia Univ., http://www.caf. wvu.edu/ forage/pastplate.htm

21. Rearte DH. 2011. El rol de las pasturas cultivadas y pastizales en el nuevo escenario de la ganadería argentina. En: Producción animal en pastoreo (Ed. INTA Balcarce), Argentina. p. 13-29.

22. Sánchez L, Iglesias A. 2009. Valoración morfológica en bovino de aptitud cárnica y razas rústicas. En: Valoración morfológica de los animales domésticos, Ed. Ministerio de Medio Ambiente, Madrid, España, p. 275-312.

23. Siles N, Vallejos A, Ferrufino A, Espinoza J. 1993. Ganancia de peso de bovinos en pastoreo en el trópico húmedo de Cochabamba, Bolivia. Pasturas tropicales, 7: 27-31.

24. Solís G. 1986. Estimación del peso corporal de ganado cebuino y sus cruces por medición del perímetro torácico. Tesis Ing. Agr. Universidad de Costa Rica. San José, Costa Rica, $85 \mathrm{p}$.

25. Villarreal VV. 2007. Correlación entre perímetro toráxico y peso vivo en la ganadería bovina del sistema doble propósito. Rev Promega 4: 19-25.

26. Wanderstock JJ, Salisbury GW. 1946. The relation of certain objective measurements to weights of beef cattle. $J$ Anim Sci 5: 264-271. 